\title{
QUANTITATIVE ASSESSMENT OF
}

\section{SCANNING-SYSTEM PARAMETERS}

\author{
A. G. Schulz, L. G. Knowles, L. C. Kohlenstein, R. F. Mucci and W. A. Yates \\ The Johns Hopkins University Applied Physics Laboratory, Silver Spring, Maryland
}

There is a recognized need to investigate radionuclide scanning on a system basis beginning with the radiopharmaceutical and the clinical problem and encompassing the instrumentation and observer performance. To be effective such investigations must be based upon quantitative measurement of system performance over a range of system-design parameters and over a range of medical problems. Although many investigators have measured the performance of discrete components, there has been no systematic investigation of the entire scanning system. This has been due largely to the difficulties associated with varying realistic phantom organs and real detector systems and the time consumed in repeating tests to compensate for statistical fluctuations. Similarly, the limited availability of appropriate data-storage and data-processing equipment has prevented effective exploration of the effects of data processing on performance.

Techniques and instrumentation have now been developed to overcome these limitations based on a combination of scanning hardware components and digital simulation to form a system for quantitatively investigating all parameters of the scanning technique. The system provides the capability for studying the effects on image interpretation due to physical characteristics of the radionuclide, characteristics of the clinical problem, characteristics of the detector and effects of various data-processing operations. In this system the input data are provided by either a digital simulation of a scanning system viewing an organ or alternatively by a scanner at the Johns Hopkins Medical Institutions scanning a phantom or a patient.

The digital simulation of a particular organ and detector provides the detector-output data in a form representing a normalized scan without statistical fluctuation noise. Stored data from these scans are then operated on by a general-purpose computer which is programmed to introduce the parameters associated with activity levels, lesion size and location, detector efficiency and scanning time, among others. After being transformed to include quantum fluctuation noise, these data represent a complete simulation of a scan. These simulation data are processed in an on-line digital computer and presented on a digitally-controlled, TV-like display. The effect of the combined parameters of the system are studied by measuring the performance of observers in detecting and characterizing lesions. The value of computed quantities such as area counts in assisting the visual evaluation of a scan can be assessed. Many different parameters can be rapidly varied, and large numbers of tests can be run so that quantitative results may be obtained consistent with the statistical nature of the problem.

The system has been used to study the effects of the density of counts, or counts per unit area, on the detectability of lesions of graded sizes in the scan image of a kidney. This density of counts in the scan image is a direct function of the activity of the radiopharmaceutical or equivalently of the total time devoted to the scan. Hence, the resultant curves of the probability of lesion detection as a function of peak counting rate provide for the first time a quantitative value on the quantity of radioactivity administered to a patient. Interpreted as a function of scan time, such curves indicate the value of scanning the patient for longer times. Finally, these same data provide a measure of scanning-system performance variations (with respect to focal lesions) to be expected from changes in other parameters that affect the counting rate, such as the change in attenuation with gamma-ray energy or the change in detector efficiency as a function of crystal size.

In the following sections the system is described, and technical data on the methods of simulation and the system hardware are provided. The methods of running the experimental tests and the test results are presented and discussed.

\section{THE METHOD}

A block diagram of the system for investigating scanning parameters is presented in Fig. 1. Two

Received Jan. 27, 1969; revision accepted Aug. 12, 1969.

For reprints contact: A. G. Schulz, The Johns Hopkins University Applied Physics Laboratory, 8621 Georgia Ave., Silver Spring, Md. 20910. 


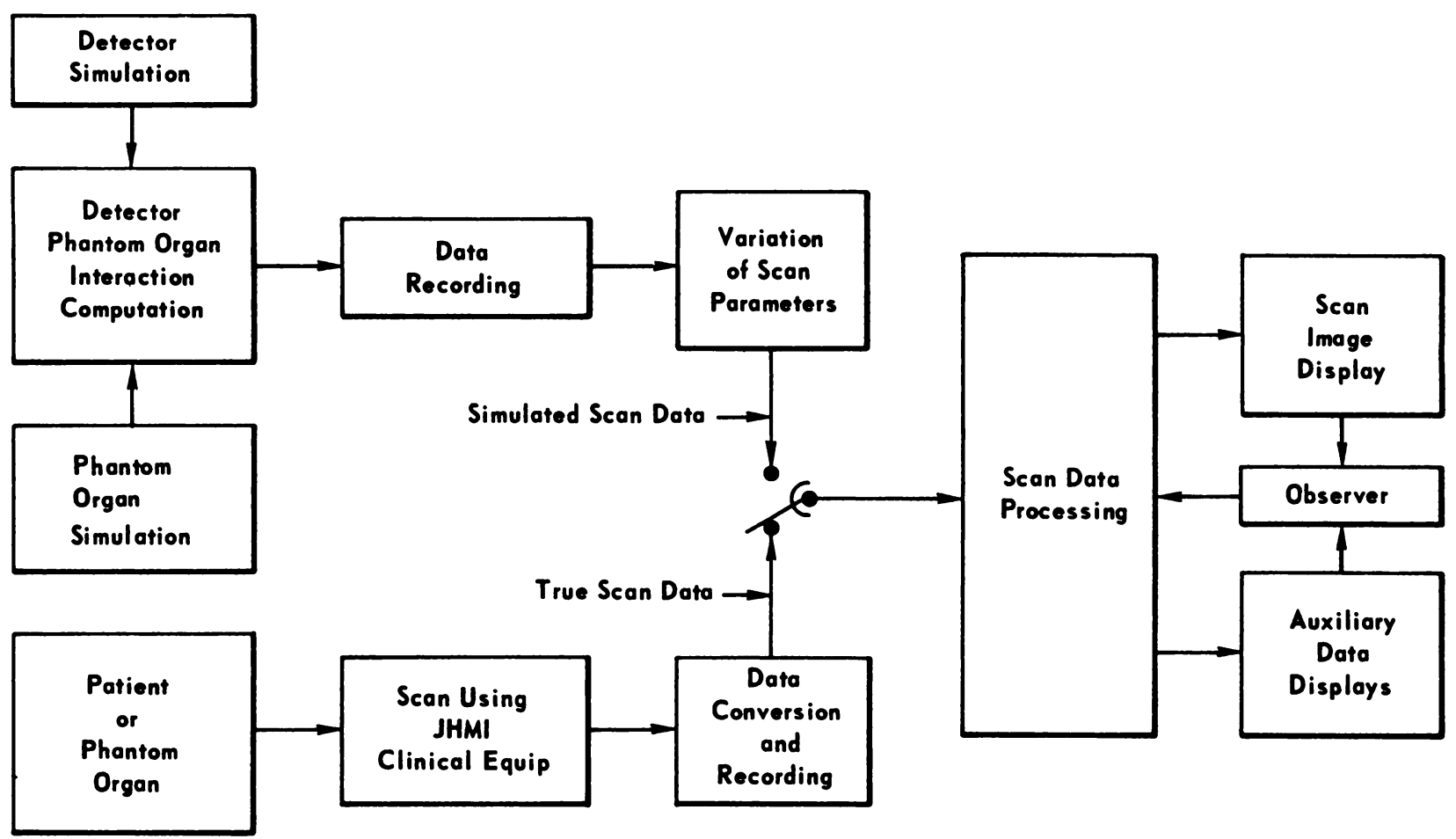

FIG. 1. Diagram of components and functions comprising scanning research system. Provision for changing from simulated scan data to clinical scan data collected at The Johns Hopkins

methods are provided for generating the map of counts measured by the detector as a function of position over the organ which constitutes the basic scan data. Simulated scan data are produced by the sequence of operations shown in the upper left portion of the figure. Input data obtained with the clinical and research scanners of the Johns Hopkins Medical Institutions are represented by the pathway in the lower left section of the figure. The on-line data processor operates on data from either input and transmits the final image to a display system designed for this purpose which maintains its own image storage. The interaction of the observer with the data-processing system and the use of auxiliary data and displays for improving the observer's ability to detect or characterize lesions is an important element in the studies proposed to be carried out with the system. The hardware and programming for initial studies are complete for the mode of operation using simulated scans. The data transform and recording system for operation of the system with patient scans or analog organ phantom scans has also been completed. The latter system provides the capability for recording the amplitude and position of each pulse and the elapsed time since the previous pulse for average rates up to $7,000 \mathrm{cps}$. In a second mode of operation the number of counts in a selected energy band in successive time intervals is recorded.

The basic data of a scan are represented by a
Nuclear Medical Center is shown schematically in figure as switching operation preceding scan-data processing and image system.

tabulation of the counts obtained by the detector as a function of its position over the distribution of radioactivity. The detailed results of this scanning process depend upon the following parameters:

1. Geometry of the organ and surrounding tissue;

2. Size, shape and location of lesions;

3. Radiation characteristics of the isotope;

4. Radioactivity levels (uptake) of the organ, lesion and surrounding areas;

5. Response characteristics of the collimatordetector;

6. Pattern and duration of the scan.

The digital simulation system, as presently developed, synthesizes the output of the radiation detector as it scans a phantom organ and provides for variation of all these parameters. To provide these data in a flexible manner at moderate cost, the simulation is divided into two parts.

The first section, performed on an IBM 7094, contains the basic interaction between the threedimensional point-spread function of the collimatordetector and the three-dimensional description of the organ phantom. The output of this section is a normalized version of the scan that would have resulted if the time taken for the scan had been long enough to completely remove the statistical fluctuations from the data and if the organ contained no lesions. The point-source response functions currently used are those actually measured in water so 
that the effects of scattering are included in the response function.* The data for the interaction of the detector with the lesion are obtained in a separate operation. Lesions of different sizes and placed at varying depths are scanned independently in this section of the simulation for later superposition on the organ/background data plane.

A detailed discussion of the techniques used in the simulation will not be attempted here since the extent and degree of detail of the three-dimensional simulation pose a complex computational problem. The basic characteristics of the program that reduce the computer storage and computation time are described.

As an aid to understanding the computational process, envision the collimator response volume divided into a three-dimensional lattice of small cubes ( $1 / 16$ of an inch on a side). Associated with each cube is a number representing the sensitivity of the collimator-detector to radioactivity in that small volume. Similarly, envision the organ and surrounding tissue volume divided into cubes of the same size; associated with each of these cubes is a number corresponding to the amount of radioactivity in that small volume. To calculate the average number of counts that would be obtained while the collimator is centered over one square $(1 / 16$ in. on a side) at the point $x, y$ in the data plane, one would superimpose the collimation volume on the organ volume with the center of the collimator at the point $x, y$, multiply the numbers in coinciding cubes and add all the products. The focal plane of the detector is held at a fixed depth $\mathrm{z}$ during the entire process, but such a summation is carried out over all values of $z$ where the organ or surrounding area has activity using collimator-detector response functions appropriate to these depths. Figure 2 shows a representation of a section at a particular value of $z$. Since we are here dealing with just two activities, we can factor the activities out of this expression and make two summations; one for collimator response cells falling within the organ and one for response cells falling in surrounding tissue. The actual values of activity associated with the organ and surrounding tissue are now free parameters to be varied without rescanning the organ. This is also true of all factors which would scale these numbers, such as scan time and crystal efficiency.

When moving the detector position from one data plane cell to the next, the summations described above change very little. Only those collimator-gain

* It is proposed in the future to calculate response functions, including the effects of scattering, from the collimator design parameters. This will allow study of the performance of collimators without first constructing them. values (cells) crossing the organ boundary contribute to the change in the summations obtained from the previous position. Therefore, it is only necessary to describe the boundaries of the organ to the computer (at each value of $z$ ), rather than indicate the coordinates of every cell within the volume.

To insert a lesion at an arbitrary position in the organ, one can subtract from the organ summation at each square affected the summation obtained at that square by scanning the lesion alone. The summations can then be multiplied by the appropriate activity scale factors and added to obtain the normalized average scan.

One of the effects of a change in the radionuclide used for the scan is the different attenuation experienced with a different gamma-ray energy. For moderate changes in gamma-ray energies, such that the difference in attenuation is significant but the change in scattering effects is not pronounced, this effect can be incorporated without rescanning the organ. This is accomplished by normalizing the point-source response function used at different depths to eliminate the attenuation effects, obtaining and saving the summations as functions of depth and finally combining the contributions from different depths with weighting functions that depend on the attenuation of the particular isotope of interest.

The basic simulated scanning of a kidney and the seven spherical leisons ranging in size from $1 / 4$ to 1 in. in dia requires $1 / 2 \mathrm{hr}$ computational time on the IBM 7094. This operation need only be repeated

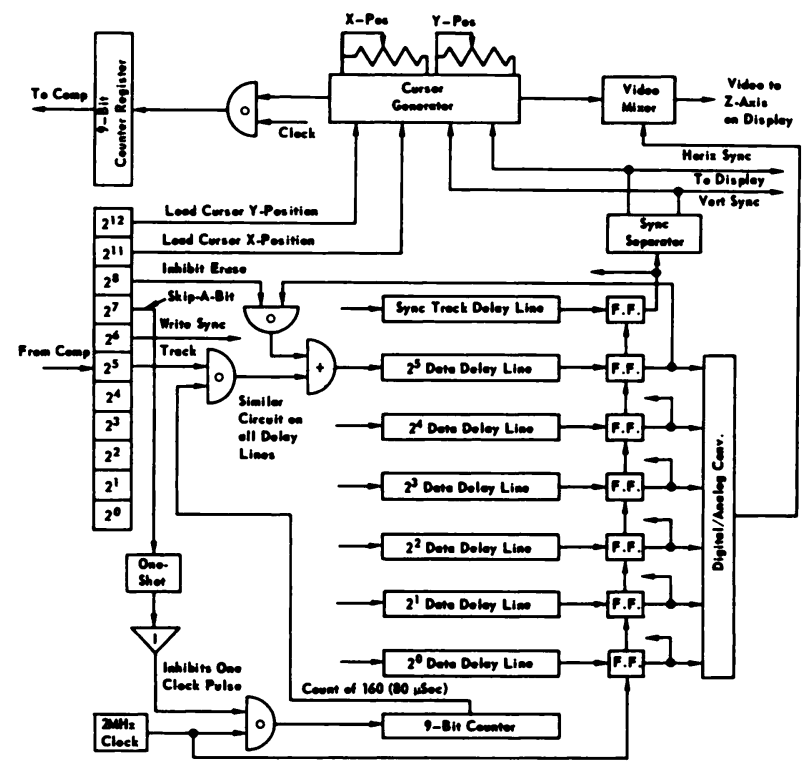

FIG. 2. Simplified illustration of computational technique used in digital simulation. Sensitivity values in collimator response function which cross organ boundary contribute to change in summation as collimator center moves from $\left(x_{1}, y_{1}\right)$ to $\left(x_{1+1}, y_{1}\right)$. 


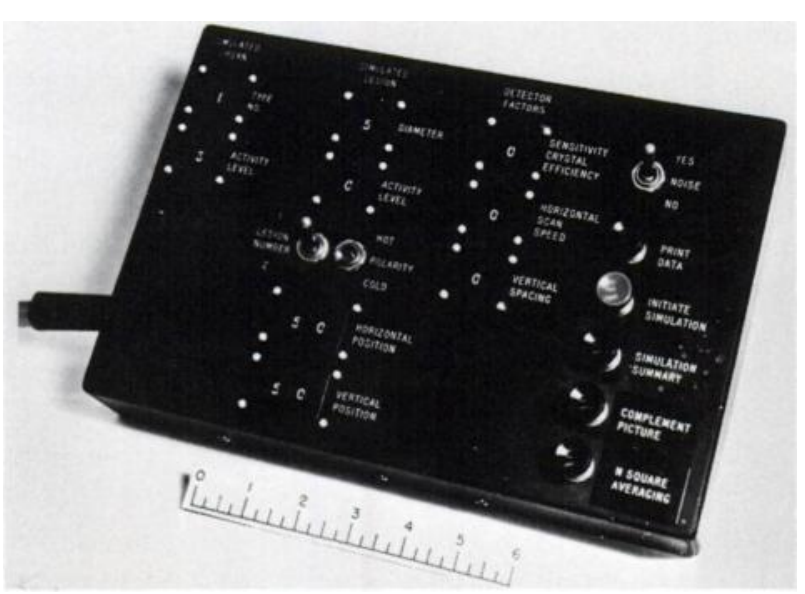

FG. 3. Scan synthesis control console which sets up on-line simulation of radioisolope scans. Console, communicating directly with computer, permits selection of organ/lesion types, numbers and location, as well as number of factors associated with detector characteristics and data-processing options.

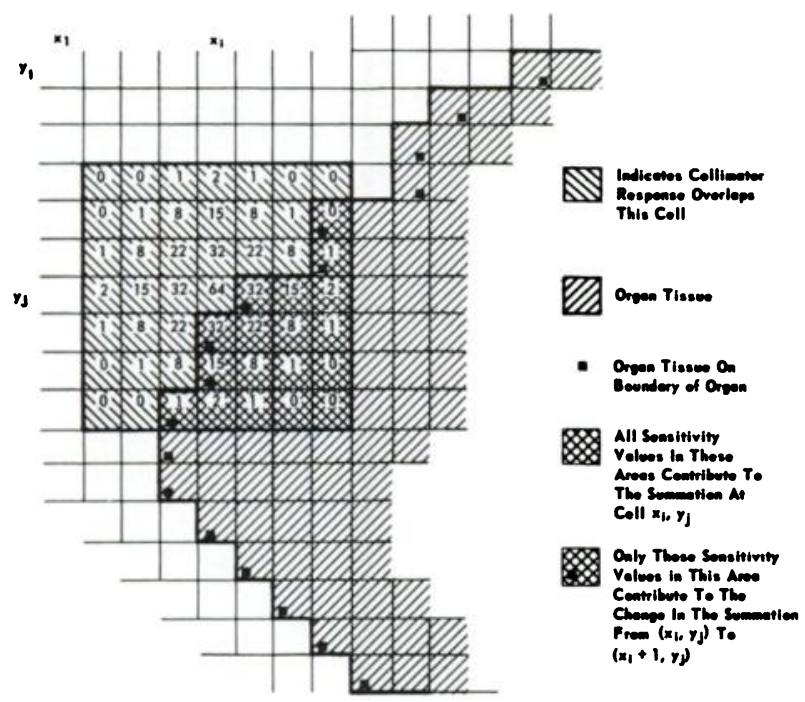

FIG. 4. Block diagram of memory system and logic networks used in image display system.

when a new collimator, a new organ or a nuclide of substantially different radiation characteristics is to be studied.

The second section of the simulation program is performed under operator control on a Univac 1230 computer. Using the scan-synthesis control console shown in Fig. 3, a set of parameters is selected and a simulated scan is computed and presented to the display system. Referring to Fig. 3, simple numerical registers are manipulated to select and read the normalized organ scan and lesion(s), to position one or two lesions in the scan and to set the detector and scan program factors. At the point when the simulation has been set up, the control to initiate the simulation is activated. The data plane mean values are computed, and a random number generator replaces the average values by quantized random numbers with a Poisson distribution. Provision is made in the control panel for the application of data processes selected by the operator. Unless it is specified otherwise in the programming, the computer automatically scales the output data to use the full 64 levels of intensity available in the display. If no special data processing is requested, the 1230 computer requires $15 \mathrm{sec}$ from the time the operator selects the values of the parameters until the finished scan is displayed.

The principal requirement of the display system is to provide an adequate resolution and dynamic range so that the display will not limit the flexibility that has been built into the scanning research system. At the same time, a reasonably low cost is considered to be a prime goal if the unit is to be directly applicable to clinical use. Additionally, it is useful for the display unit to provide the means, within itself, to regenerate the picture so that the system computer is not tied up performing this repetitious function.

The unit that was developed incorporates a 240,000-bit, 20,000- $\mu$ sec-long magnetostrictive delay-line memory which refreshes the $160 \times 250$ matrix of picture elements, ${ }^{*} 50$ times/sec. (1). A basic block diagram of the display system is given in Fig. 4.

The 17-in., TV-like picture containing the 40,000element data plane is provided with the intensity at each point variable in 64 steps, from approximately 1.3 to $8.2 \mathrm{ft}$-lamberts. The six bits of intensity code for each particular picture element are loaded into and travel down an array of six delay lines operating in parallel. All of the bits arrive together at the reclocking station, where slight irregularities in the transit time are subtracted out and the reclocked word sent back to the beginning of the line for recirculation. Also at this point, the digital data is converted on-the-fly to analog video by the $D / A$ ladder network. This video then modulates the intensity on a cathode-ray-tube screen.

An additional timing delay line contains the horizontal and vertical synchronization signals so that the proper output data matrix is developed on the cathode-ray tube. The vertical sweep is the fast sweep; the period of the sawtooth generator is 80 $\mu \mathrm{sec}$. Data changing at the $2-\mathrm{MHz}$ clock rate supplies the 160 vertical elements per line. The horizon-

\footnotetext{
* Current renal images use only $80 \times 250$ of these elements.
} 
tal sweep generator runs at the frame rate of once per $20,000 \mu \mathrm{sec}(50 \mathrm{frames} / \mathrm{sec})$. At $80 \mu \mathrm{sec} / \mathrm{line}$ this then supplies 250 horizontal elements.

An electronic cursor feature has been built into the system to allow the operator to effectively communicate with the controlling computer. The cursor takes the form of a pair of orthogonal dark lines, each of which is one-element $(1 / 16$-in.) wide. The operator may adjust the $x / y$ position of the cursor by using a set of two independent controls. Special electronic circuitry senses this position and sends the data back to the computer where they may be correlated with other operator-selected commands that require a position input.

The cursor has been most useful in defining a special area for investigation by an auxiliary display. This special display gives the intensity profile as a function of distance along a single line in the display. The coordinate of this cut through the picture is defined by the cursor position on the main display. As yet, we have not evaluated the diagnostic value of the intensity profile.

\section{EXPERIMENTS}

Initial experiments have been carried out with a digital simulation of a kidney scanned with a focusing collimator having a $3 / 8$-in. radius of view designed for the 8-in. Hopkins scanner (2). The computation was carried out with the focal plane of the collimator at the midline of the organ. The collimator was designed for $360-\mathrm{keV}$ gamma rays of ${ }^{131}$ I using the equations of Beck (3). It has an efficiency of 0.034 $\mathrm{cm}^{-2}$ and a calculated penetration of $5 \%$. For the present simulation the response function and efficiency of the detector were measured in a phantom with ${ }^{197} \mathrm{Hg}$. The attenuation of the gamma rays was compensated for in generating the point-spread functions at various depths and in checking detector efficiency. A set of spherical lesions with nominal diameters from $1 / 4$ to 1 in. in $1 / 8$-in. steps was computationally scanned in an identical manner. The lesions were centered at the focal plane of the collimator.

The simulated test scans were set up to cover the range of conditions and counting rates corresponding to clinical experience with the particular scanner being simulated. This selection of test parameters will permit the simulated images to be directly checked with those of actual scans with the use of the newly completed data recording system. The initial scans simulate a 10-min scan (per kidney) with counting rates at the maximum position over the kidney ranging from 3,000 to $48,000 \mathrm{cpm}$. For this same scanner the clinical experience generally ranges from 15,000 to $25,000 \mathrm{cpm}$ with an administered dose of $300 \mu \mathrm{Ci}$ of ${ }^{197} \mathrm{Hg}$-labeled chlormerodrin. Since many scanning units in use have detectors with lower efficiency than the 8-in. detector crystal unit being simulated here and since smaller doses of radiopharmaceuticals are given in some procedures, the lower range was set to cover such cases. The upper portion of the counting-rate range was set high enough to demonstrate the gains which might be made by several ways of increasing counting rates including the use of improved pharmaceuticals-permitting higher activities and the gains obtained from the use of longer scan times.

The method of testing for lesion detectability is based on test sessions in which several observers are presented with the images of 70 simulated scans at a single organ activity level but with randomly varying lesion sizes and positions. In each test set the first five scans are orientation scans with one example involving no lesion and four examples in which the position of the lesion is made known to the observer so that he may become accustomed to the general characteristics of the scans at that activity level and to the test environment. ${ }^{*}$ In the next 65 tests between 15 and 20 tests have no lesion present. The observer is asked to decide whether or not he thinks there is a lesion; if so, he is asked to define its location and to assign a confidence factor to that judgment. The confidence factors were described as suspected lesion, probable lesion and very probable lesion. After each test frame there is a feedback frame in which the presence of a lesion and its location are indicated in the scan image. The observers mark their evaluation of the scan on score sheets during the test frame and then grade themselves by marking the correctness of the observations during the feedback frame. The tests are set up in advance on magnetic tape and played back under computer control with the test frame presented for $20 \mathrm{sec}$ and the feedback frame presented for $5 \mathrm{sec}$. With a 5-sec rest between frames, each 70-test sequence takes approximately $3 / 4 \mathrm{hr}$. The positions of the lesions are approximately uniformly dense throughout the central region of the organ. The number of times each lesion size occurs in a set was determined from earlier unpublished tests to provide better statistical confidence levels in regions where the results are most interesting. A photograph of a typical simulated scan image as displayed to the test observers is shown in Fig. 5.

\footnotetext{
* The level at which the screen gave adequate dynamic range made it desirable to conduct the tests in a room with dimmed lights. Screen luminance was adjusted to approximately $8 \mathrm{ft}$-lamberts in the brightest point of the image. A new display unit permits operation at higher luminances and greater dynamic range.
} 
A sequence of four test series at each of six maximum counting rates was conducted with four specific observers participating throughout the entire program. The observers in these tests are engineers and physicists who have acquired a continuing interest in the scanning field and who are familiar with scan data and several forms of data presentation. It is planned to run tests with clinically oriented medical observers. Each of the four observers took 1,200 tests containing lesions, leading to a total of 4,800 possible detections in the entire series.

\section{RESULTS}

The basic data from these tests are shown plotted in Fig. 6A, B and $\mathrm{C}$. In each figure the experimentally derived probability of detection of the lesions is plotted as a function of the maximum counting rate over the organ for various lesion diameters. In the successive figures the confidence rating assigned to the detection is varied. The confidence ratings assigned to a detection were: suspected lesion, probable lesion and very probable lesion. In Fig. 6A all correctly located detections are counted including all confidence ratings. In Fig. 6B only correct detections assigned a confidence rating of

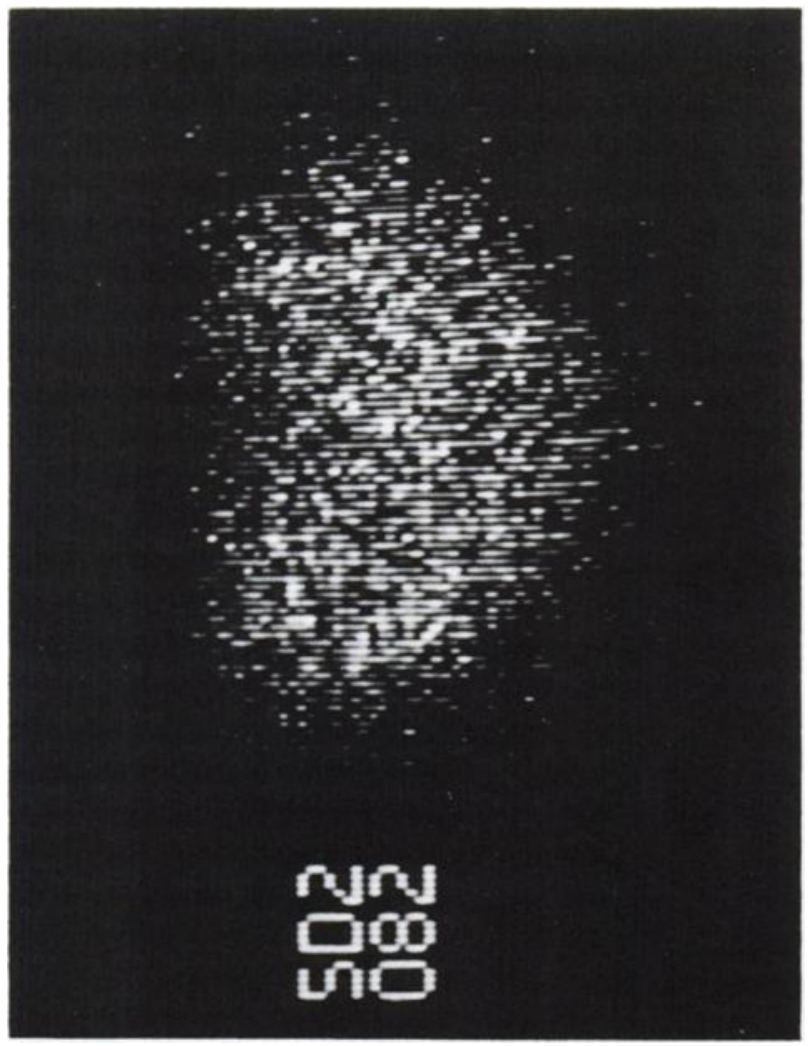

FIG. 5. Photograph of typical scan image showing a K-in.dia lesion in lower lobe of kidney. Scan shown corresponded to peak counting rate of $12,000 \mathrm{cpm}$. This size lesion was detected about $75 \%$ of time at this counting rate.

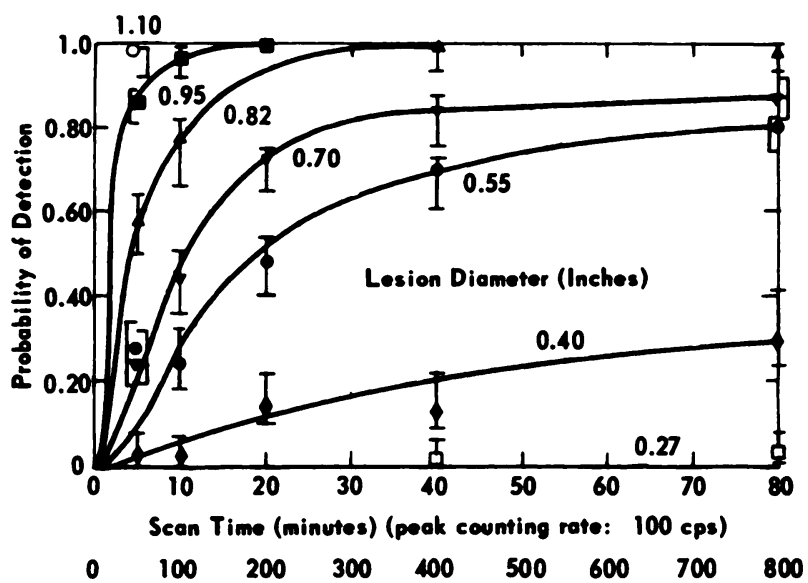

A Peak Counting Rote (counts per second) (scon time: 10 minutes)

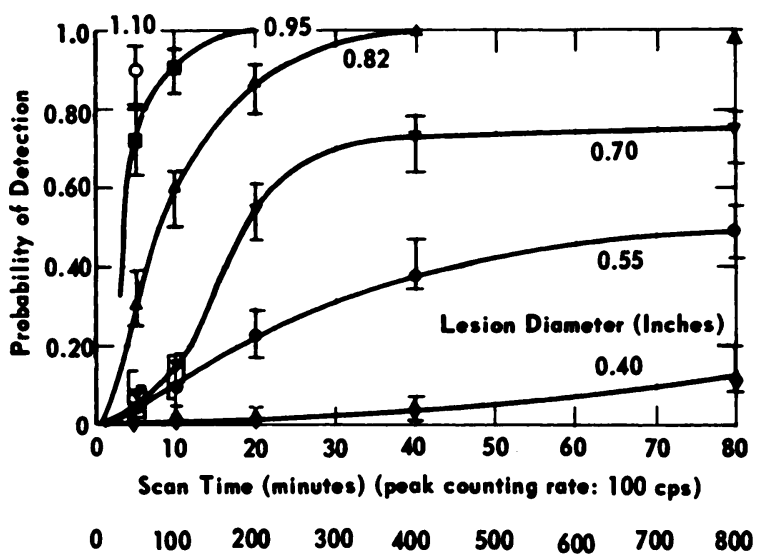

(3) Pook Counting Rote (counts per socond) (scan time: 10 minutes)

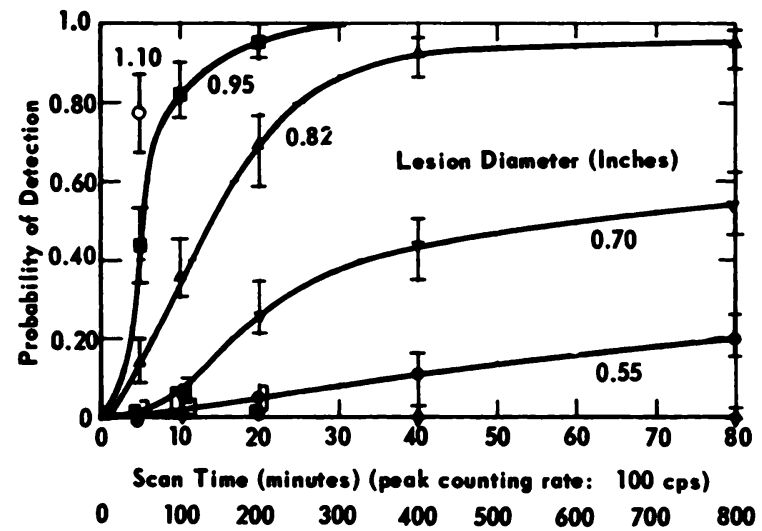

Pook Counting Rate (counts per second) (scan time: 10 minutes)

FIG. 6. Probability of defection for cold lesions of various diamefers plotted as function of maximum counting rate measured over kidney (assuming fixed scan duration) and, equivalently, as function of sean duration (for a fixed peak counting rate). A: Correctly located suspected lesions, probable lesions and very probable lesions are all counted as defections. B: Correctly locafed probable lesions and very probable lesions are counted as defections. Cs Correctly located very probable lesions are counted as defections.

probable or very probable are scored. Correctly located detections assigned a rating of very probable are scored in Fig. 6C. 
A more quantitative estimate of the significance of confidence ratings is indicated by the analysis of the errors in each category. When the rating of suspected lesion was assigned, the detection was correct only $50 \%$ of the time averaged over the four . observers. The other $50 \%$ of these suspected lesion detections were false alarms with respect to this category. The spread among observers was from $40 \%$ correct to $55 \%$ correct. When the observers assigned the next higher rating of probable lesion to a detection they were correct $85 \%$ of the time, and detections assigned as very probable were correct $99 \%$ of the time.

The data plotted in Fig. 6A, B and $\mathrm{C}$ are for simulated scans covering a range of activities corresponding to maximum counting rates of 50,100 , 200,400 and $800 \mathrm{cps}$, assuming the parameters of the Hopkins 8-in. crystal scanner (2). The errors shown in Fig. 6A, B and C are the $95 \%$ confidence limits. Another set of data was taken in the same manner as these but with the activity assumed to be so high that there is no quantum fluctuation noise associated with the scan. Performance in this case is presumed to define the limit of observer performance imposed by the response of the observer's eye, the resolution of the detector (including all penetrations and scattering effects) and the variation in the spatial distribution of activity resulting from unknown details of the structure of the organ. For this image-noise-free case the detection probabilities obtained are presented in Table 1 for the three groupings of confidence ratings previously discussed.

\section{DISCUSSION OF THE RESULTS}

The results of the measurements of observer performance in detecting lesions in simulated scans as the maximum counting rate or total count is varied may be interpreted in terms of several system variables which affect the count without changing other critical parameters. The counting rates considered in the study span a range of values which cover minimum current clinical practices on the low end to counting rates in excess of those achieved with current pharmaceuticals and instruments on the upper end. Two basic ramifications of the data will be discussed: the relative effects on performance of certain system variables such as radionuclide dose, scan time or detector efficiency and the performance predictions for other systems on the basis of the performance of the system used in this study.

For the system tested the relative variation of performance with changes in administered dose or effective organ uptake may be read directly from the curves of Fig. 6A, B and $\mathrm{C}$ using the lower abscissa scale since the peak counting rate $C_{n \text { o }}$ plotted there is linearly related to the specific activity of the organ and, therefore, to the administered dose and organ uptake. The relative variation of performance with the total time for the scan may be read directly from the upper abscissa scale. Therefore, for this type of scan these curves provide a quantitative estimate of the improvement in lesiondetection performance that a clinician could expect from an increased scan time or an increased dose of a given radiopharmaceutical or an improved pharmaceutical which provides a higher effective count in the scan.

TABLE I. MEASURED PROBABILITY OF DETECTION AND LIMITS FOR 95\% STATISTICAL CONFIDENCE FOR VARIOUS SIZE LESIONS MEASURED FOR IMAGES WHICH CONTAINED NO STATISTICAL FLUCTUATION NOISE

\begin{tabular}{|c|c|c|c|c|c|c|}
\hline \multirow{3}{*}{$\begin{array}{l}\text { Lesion } \\
\text { diam- } \\
\text { eter } \\
\text { (in.) }\end{array}$} & \multicolumn{6}{|c|}{ Lesion probability of defection } \\
\hline & \multicolumn{6}{|c|}{ Observer confidence rating: } \\
\hline & \multicolumn{2}{|c|}{$\begin{array}{l}\text { Suspected, } \\
\text { probable, } \\
\text { and very } \\
\text { probable }\end{array}$} & \multicolumn{2}{|c|}{$\begin{array}{l}\text { Probable } \\
\text { and very } \\
\text { probable }\end{array}$} & \multicolumn{2}{|c|}{$\begin{array}{c}\text { Very } \\
\text { probable }\end{array}$} \\
\hline 0.27 & 0.39 & $\begin{array}{l}0.45 \\
0.33\end{array}$ & 0.12 & $\begin{array}{l}0.15 \\
0.08\end{array}$ & 0.04 & $\begin{array}{l}0.07 \\
0.02\end{array}$ \\
\hline 0.40 & 0.98 & $\begin{array}{l}0.99 \\
0.95\end{array}$ & 0.88 & $\begin{array}{l}0.92 \\
0.83\end{array}$ & 0.61 & $\begin{array}{l}0.66 \\
0.53\end{array}$ \\
\hline 0.55 & 0.99 & $\begin{array}{l}1.00 \\
0.96\end{array}$ & 0.99 & $\begin{array}{l}1.00 \\
0.96\end{array}$ & 0.99 & $\begin{array}{l}1.00 \\
0.97\end{array}$ \\
\hline 0.70 & 1.00 & & 1.00 & & 1.00 & \\
\hline
\end{tabular}

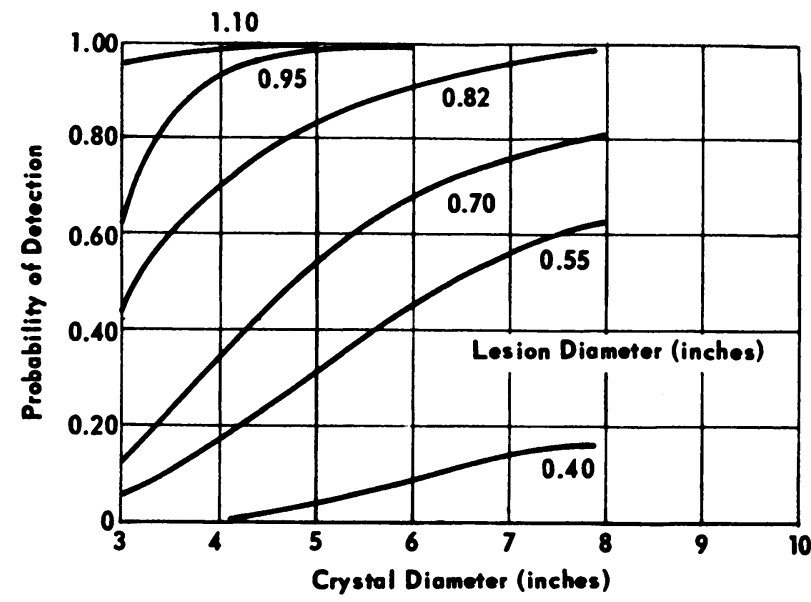

FIG. 7. Probability of defection plotted as function of defecfor erystal diameter for various lesions sizes. These data are referenced to administered dose and uptake giving peak counting rate of 300 cps with Hopkins 8-in. crystal scanner. 
Another system parameter which controls counting rate is the diameter of the detector crystal. The relative variation of system performance with detector crystal size for regular focusing collimator detectors is plotted in Fig. 7. In this figure the data are referenced to the performance of the 8-in. crystal detector at a peak counting rate of $300 \mathrm{cps}$ which is typical of current clinical experience with this unit in kidney scanning. These curves provide an estimate of performance for systems with detector crystals of smaller diameters. It is necessary to keep in mind that the results are for a relatively shallow organ located at the focal plane of the focusing collimator. The results are expected to be less favorable for the larger crystals applied to a deep organ with a lesion displaced from the focal plane of the detector.

Experience with other systems may be correlated with results obtained here to estimate performance expectations for these systems. The potential gains in performance resulting from steps taken to achieve more photon data per scan in any system may be assessed. In extrapolating the results to estimate the performance of other systems, the equivalence of variations in radiopharmaceutical, instrument parameters or clinical use which lead to scans containing the same number of photon counts must be recognized. All of the results reported here are based on a simulated scan time of 10 min for one kidney (the total area scanned is approximately 37 in. $^{2}$ ). Within limits to be discussed below, the same performance reported here at a maximum counting rate $C_{m o}$ and scan time $T_{0}\left(T_{0}=10 \mathrm{~min}\right)$ should apply to a situation with another system in which the maximum counting rate is $C_{m x}$ provided the scan time $T_{x}$ in the latter case is such that

$$
\mathrm{T}_{\mathrm{x}} \mathrm{C}_{\mathrm{mx}}=\mathrm{T}_{\mathrm{o}} \mathrm{C}_{\mathrm{mo}}=10 \mathrm{C}_{\mathrm{mo}}
$$

where $\mathrm{C}_{\mathrm{mo}}=$ maximum counting rate in present study as shown in Fig. 6A, B, and C in units of counts per second.

$T_{0}=$ total scan time in present study which is equal to $10 \mathrm{~min}$.

$\mathrm{C}_{\mathrm{mx}}=$ maximum counting rate of system to be compared in units of counts per second.

$T_{x}=$ total scan time for system to be compared in minutes.

Provided the organ thickness is similar to that of a kidney, and provided the resolution characteristics are about the same as those of the collimator used here, the above result is essentially independent of the cause of the change in counting rate including changes in detector crystal size, in administered dose or in uptake of the organ.

\section{CONCLUSIONS}

A system has been developed with which quantitative evaluation of scanning-system effectiveness in terms of the lesion detection and location performance of observers is practical. The process of scanning a three-dimensional nuclide distribution with a radionuclide imaging system has been digitally simulated. With this simulation it is possible to vary in a flexible manner the parameters associated with the radiopharmaceutical, the organ and abnormalities, the collimator-detector, the data processing and the display. A simulated scan including the statistical fluctuations can be produced and displayed on a cathode-ray viewing screen or recorded on magnetic tape at a rate of $3 / \mathrm{min}$. This speed permits us to reach quantitative conclusions about the effects of variations in the scanning system on a basis of a statistically significant number of scans.

In the study reported here, scans of a kidney containing ${ }^{107} \mathrm{Hg}$ were simulated. The parameters varied in this study were the count density in the image and the size of the spherical lesions. Changes in count density were related to corresponding changes in the activity within the organ, in the scan time and in the crystal size. The frequency with which the observer detected the abnormalities under the various conditions is reported for several levels of observer confidence. The curves provide a measure of the value of increased count density and of the relative detectability of different-size lesions in a shallow organ such as the kidney.

\section{ACKNOWLEDGMENT}

The authors wish to thank Henry N. Wagner, Jr. for his guidance and support in carrying out this work. This work was aided by U.S. Public Health Service Grant GM 10548.

\section{REFERENCES}

1. Knowles, L. G. and Yates, W. A.: A medical display for computer processed data: An approach to a lowcost clinical unit. 20th Annual Conference on Engineering in Medicine and Biology, Boston, Mass., Nov. 1967.

2. McAfee, J. G., Mozley, J. M., Natarajan, T. K., Fueger, G. F., WAGNer, H. N.: Scintillation scanning with an $8^{\prime \prime}$ diameter sodium iodide (T1) crystal. J. Nucl. Med. $7: 521,1966$.

3. BECK, R. N.: Collimators for radioisotope scanning systems. In Medical Radioisotope Scanning, vol. 1, IAEA, Vienna, 1964, p. 211. 